\title{
Dissociative double ionization of acetylen in strong laser field
}

\author{
Atia-tul-noor ${ }^{1}$, Han $\mathrm{Xu}^{1}$, Xiaoshan Wang, R. T. Sang ${ }^{1}$, Igor Litvinyuk ${ }^{1,2}$ \\ ${ }^{1}$ Centre for Quantum Dynamics and Australian Attosecond Science Facility, \\ Griffith University, Nathan Campus, QLD 4111, Brisbane Australia \\ ${ }^{2}$ E-mail: i.litvinyuk@griffith.edu.au
}

\begin{abstract}
Summary (35 words)
\end{abstract}
We studied the dynamics of dissociative double ionization of acetylene using pump-probe technique with few-cycle laser pulses and Reaction Microscope detection system.

Keywords: dynamics, pump-probe, double ionization

\section{SUMMARY}

Acetylene is one of the symmetric organic and polyatomic molecules to study the photo-initiated processes. Important features of intermolecular dynamics of acetylene have been revealed over many years through numerous spectroscopic studies. More recently, the availability of strong laser field has led to exciting studies of the ionization and breakup dynamics of acetylene. Using the COLTRIMS (cold target recoil ion momentum spectroscopy) technique [1] in combination with few-cycle laser pulse and with pump probe technique we mapped different dissociation pathways. The ionization in acetylene induces many break-up channels with the most interesting being the deprotonation, symmetric and asymmetric dissociation channels for two body breakup [2]. Time evolution of nuclear wave packets, isomerization, elimination and charge transfer process in molecule are studied under unrivalled conditions.

The laser pulse had duration of $6 \mathrm{fs}$ with a central wavelength of $800 \mathrm{~nm}$. Pump and probe pulses were produced with Mach-zehnder interferometer. The delay between the pump and probe pulses is controlled by computerised translational stage in the probe arm of the interferometer. The pump and probe pulses were overlapped in space and time at zero delay and directed into the COLTRIMS main chamber. Inside the chamber the laser beams were tightly focused by a silver coated spherical mirror of focal length $75 \mathrm{~mm}$ onto the supersonic jet. Pump power was $15 \mathrm{~mW}$ and probe power was $6 \mathrm{~mW}$. Probe power was set in such a way that no $\mathrm{C}_{2} \mathrm{H}_{2}^{++}$ions produced with probe alone. The ions were extracted onto a time-and position-sensitive channel plate detector (Roentdek) by a uniform electric field. The ionic products such as $\mathrm{H}^{+}, \mathrm{CH}^{+}, \mathrm{C}^{+}, \mathrm{CH}_{2}^{+}, \mathrm{C}_{2} \mathrm{H}^{+}, \mathrm{C}_{2} \mathrm{H}_{2}^{++}, \mathrm{C}_{2} \mathrm{H}_{2}^{+}$were detected. To analyze the correlated ion pairs, we only selected the pairs of fragments with total momentum close to zero and they are separated by the PIPICO (Photo ion photo ion coincidence) method.

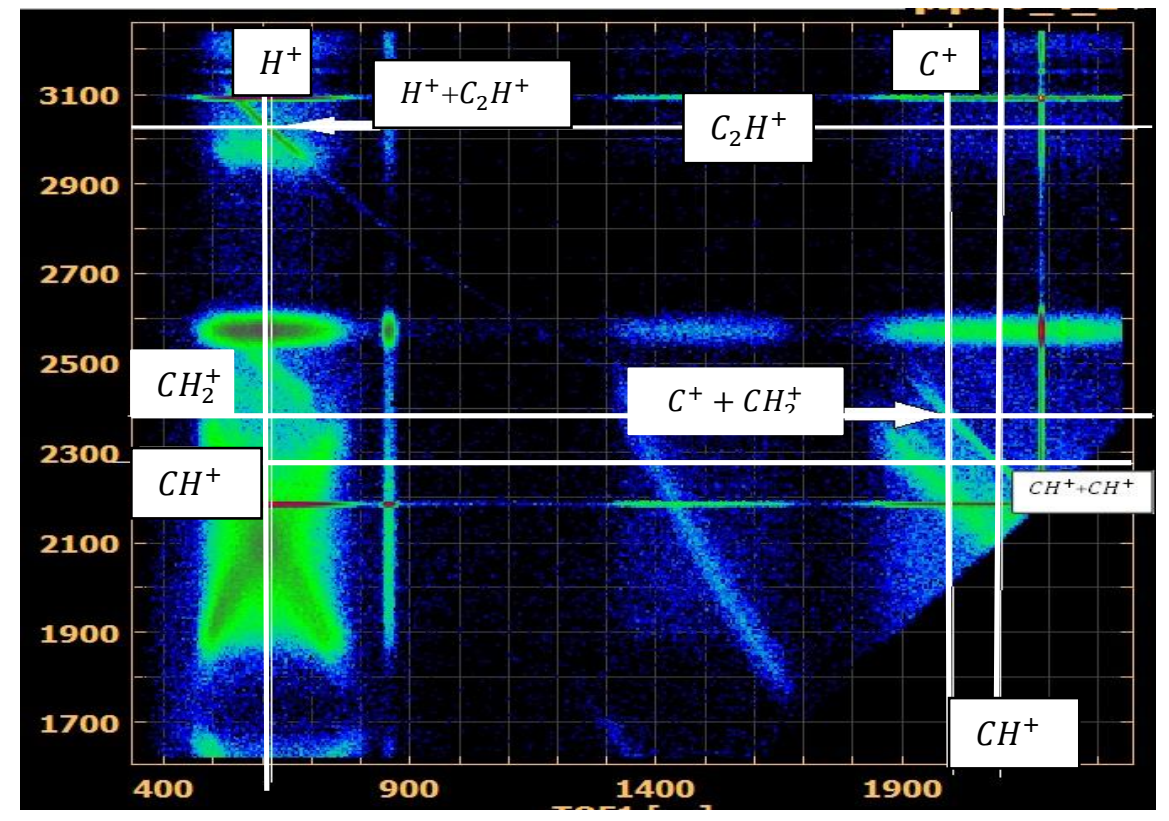

Figure 1: PIPICO Spectrum for dissociation of acetylene 
Figure1shows a PIPICO spectrum where diagonal lines represent one pair of ion fragments, with X and Y values representing the two corresponding times of flight. The sharp lines correspond to real coincidences with near zero total momentum for each break up channels observed in the experiment. Following two body breakup channels were selected for the double ionization of acetylene

$$
\begin{array}{ll}
\mathrm{C}_{2} \mathrm{H}_{2}^{++} \rightarrow \mathrm{H}^{+}+\mathrm{C}_{2} \mathrm{H}^{+} & \text {P Channel (deprotonation channel) } \\
\mathrm{C}_{2} \mathrm{H}_{2}^{++} \rightarrow \mathrm{CH}^{+}+\mathrm{CH}^{+} & \text {A Channel (symmetric channel) } \\
\mathrm{C}_{2} \mathrm{H}_{2}^{++} \rightarrow \mathrm{C}^{+}+\mathrm{CH}_{2}^{+} & \text {V Channel (asymmetric channel) }
\end{array}
$$

The kinetic energy release integrated over all delay times for the above mentioned fragmentation channels. The KER distributions for channel $\mathrm{P}$ and channel $\mathrm{V}$ exhibits maxima at $3.1 \mathrm{ev}$ and $3.5 \mathrm{ev}$ respectively also KER spectra are very nearly single valued for these channels and different than previously reported measurements [3]. The deprotonation channel shows the largest yield as compared to other two channels.

We also studied the behavior of dissociating molecule as a function of delay and observed time dependent features for different dissociating channels. The spectra in figure 2 shows the KER and Yield evolution of deprotonation and asymmetric and symmetric channels respectively

a)
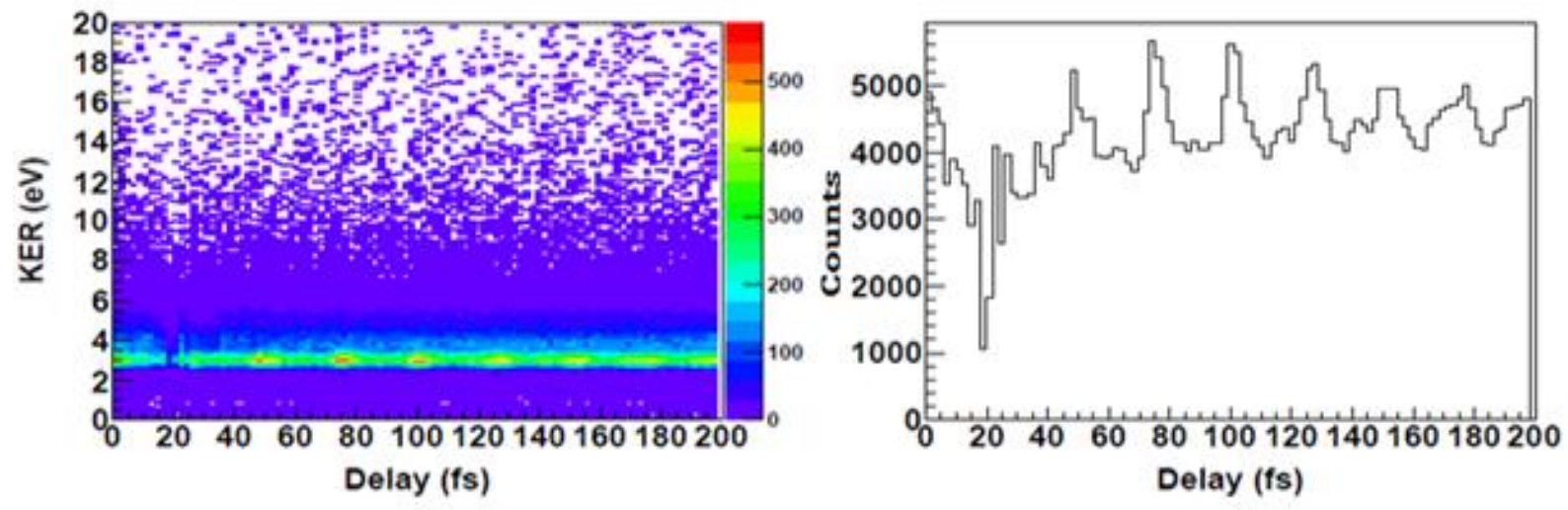

b)
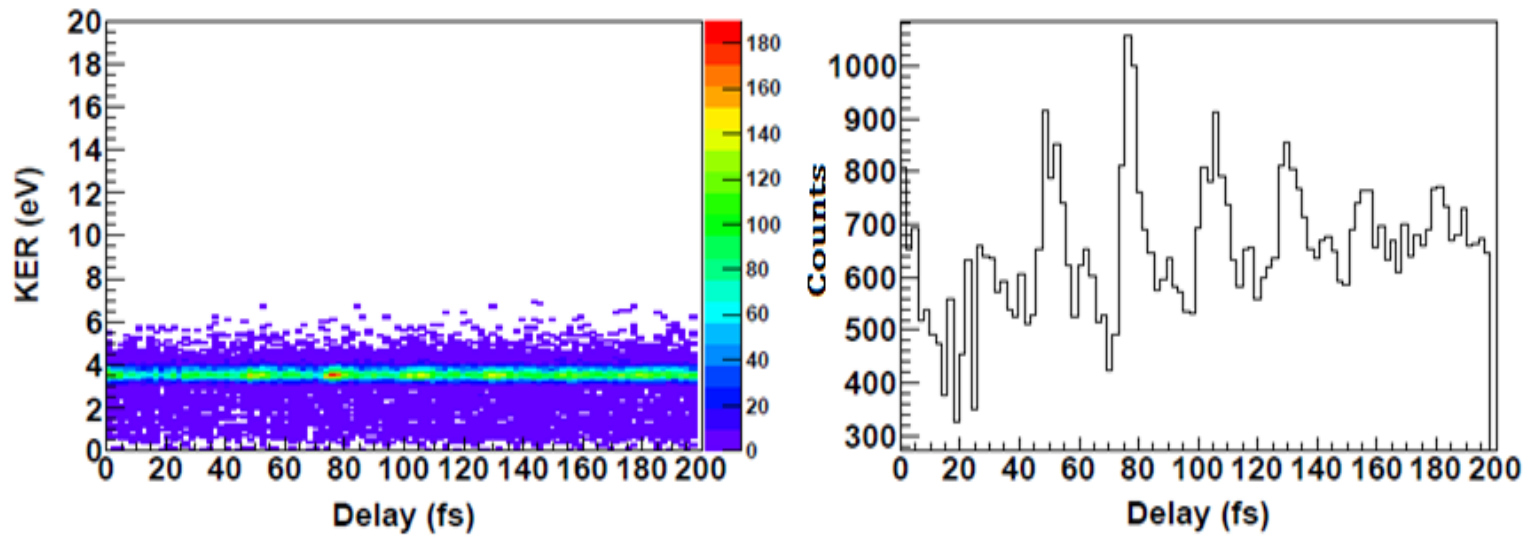
C)
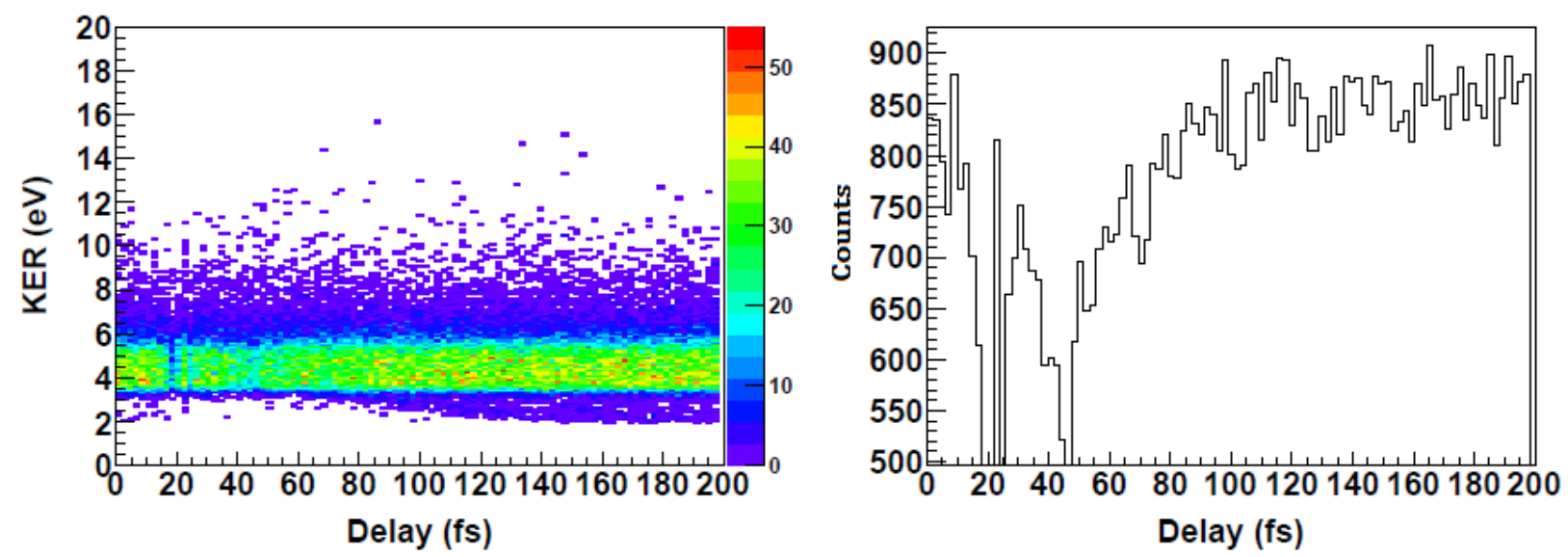

Figure2: KER vs time delay and ion yield for a) deprotonation channel, b) asymmetric channel, c) symmetric channel

The oscillatory structures in these spectrums are dominant for channel $\mathrm{P}$ and channel $\mathrm{V}$, while it would be tempting to seek physics in these behaviours; we suspect these results from vibrational modes that can be involved either C-C stretch or one of the bent modes. Since the probe cannot doubly ionize, we assume that the pump launched a vibrational wave packet on the 1+ charge state and the probe promotes this wavepacket to double ionization, on a electronic state that is dissociative along $\mathrm{C}-\mathrm{H}$ coordinate [4]. Additionaly the KER of $\mathrm{H}^{+}$is constant with time, the $\mathrm{C}-\mathrm{H}$ bond length is not changing significantly with time. We observed a strong-field ionization probability modulation by a change in molecular geometry (vibration).

The present experimental scheme demonstrates the attractive perspectives and potential applications for the timeevolution of nuclear wave packets, molecular dissociation, isomerization, elimination and charge transfer processes in molecules in strong laser field.

\section{Reference.}

[1] Han Xu, Tian-Yu Xu, Feng He, D. Kielpinski, R.T.Sang and I.V. Litvinyuk, "Effect of nuclear mass on carrier-envelope-phase controlled electron lacalization in dissociating molecules," Phys. Rev.A, vol. 89, p. 041403(R), (2014).

[2] R. Thissen, J. Delwiche, J. M. Robbe, D. Duflot, J. P. Flament and J. H. D. Eland, "Dissociations of the ethyne dication," J. Chem. Phys, vol. 99, 1993.

[3] A S Alnaser, I. Litvinyuk, T Osipov, B Ulrich, A Landers, E Wells, C M Maharjan, P Ranitovic, I Bochareva, D Ray and C L Cocke, "Momentum-imaging investigation of the dissociation of D2+ and isomerization of acetylent to vinylidene by intense laser short pulse," Phys B Atomic Molecular and Optical physics, vol. 39, 2006.

[4] T. Osipov, T N Rescigno, S Miyabe, T Jahnke, A S Alnaser, M P Hertlein, O Jagutzki, L Ph H Schmidt, M Schoffler, L Foucar, S Schossler, T Havermeier, M Odenweller, S Voss, B Feinberg, A L Landers, M H prior, R Dorner, C L Cocke and A Belkacem, "Fragmentation pathways for selected electronic states of the acetylene dication," J.Phys, B (091001, vol. 41, 2008. 